\title{
Measurement of Membrane Displacement Using a Motionless Camera
}

\author{
K. MURAWSKI* \\ Military University of Technology, Institute of Teleinformatics and Automatics, \\ S. Kaliskiego 2, 00-908 Warsaw, Poland \\ (Received May 12, 2014)
}

\begin{abstract}
The paper presents a technique for measuring membrane displacements with one motionless camera. The method relies on measuring the distance to an object based on one image obtained from a motionless camera with a fixed-focus lens. It is proven that the used technique allows monitoring the frequency and amplitude of vibration. The tests were performed for the oscillation frequency in the range from $0.5 \mathrm{~Hz}$ to $6 \mathrm{~Hz}$ and deviations from the neutral position in the range of $\pm 3 \mathrm{~mm}$.
\end{abstract}

DOI: 10.12693/APhysPolA.128.10

PACS: 06.30.Bp, 07.05.Pj, 07.07.Df

\section{Introduction}

Measuring the membrane displacement, non-contact measurement in particular, has many applications $[1,2]$. Apart from this it still generates a lot of problems, especially in difficult conditions [3]. The parameters of the membrane displacement are determined then by measuring other physical quantities. For this reason attempts are made to use fibre pressure sensors [4,5], piezofoils [3], the Helmholtz resonator [6] and optical sensors [7-9]. When the dimensions of the sensor do not impose significant limitations, fluorescence videogrammetry [10] can be used as well as the method described in [11].

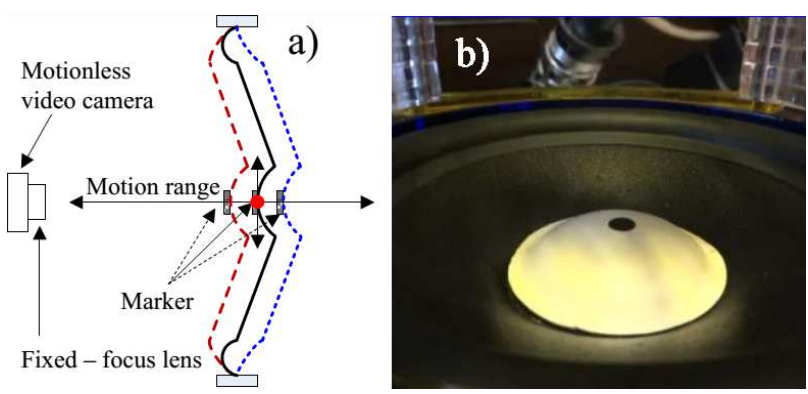

Fig. 1. Displacement model (a), view of the monitored membrane (b).

This paper presents a new approach to measuring the displacement parameters of the membrane, Fig. 1. The measurement was implemented with a stationary IR camera equipped with a fixed-focus lens and using a measurement technique based on digital image processing [12-15]. The displacement of the membrane was determined by observing the changes in the size of the marker placed on the surface. The size of the marker was measured using the method presented in [16].

*e-mail: k.murawski@ita.wat.edu.pl
The searched for membrane displacement parameters were determined in real-time based on a single photo for measuring the amplitude and the sequence of photos when the vibration frequency was measured. The tests were performed in the system presented in Fig. 2. The model in Fig. 2 was equipped with a membrane with a rubber surround, Fig. 1b. The membrane was connected to a voice coil, which was placed in a constant magnetic field. The movement of the membrane was generated by applying DC or AC voltage of a known shape, amplitude and frequency to the connectors of the coil.

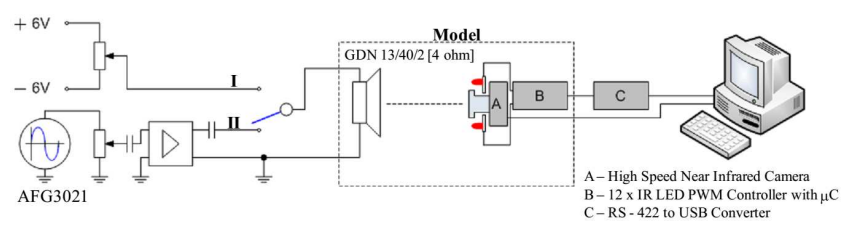

Fig. 2. The system for testing the parameters of the membrane displacement.

\section{Measurement system configuration}

The frequency and the displacement of the membrane were measured with a motionless camera equipped with a fixed-focus lens. The measurement was done using the technique described in [16]. The study was carried out in a setup as shown in Fig. 2.

The main element of the system is the model presented in Fig. 3a. The model was developed based on the membrane of a TONSIL GDN 13/40/02 woofer with parameters: rated frequency range $90-5000 \mathrm{~Hz}$, rated impedance of $4 \Omega$, voice coil resistance is $3.45 \Omega$, a total moving mass of $8.4 \times 10^{-3} \mathrm{~kg}$, a force factor of $5.03 \mathrm{Tm}$, a voice coil height of $9 \mathrm{~mm}$, a height of the magnetic gap of $6 \mathrm{~mm}$, a piston area of $85 \mathrm{~cm}^{2}$. The model enabled the safe moving of the woofer membrane to its rest relative position in the range of $\pm 3 \mathrm{~mm}$. The membrane is provided with a marker, Fig. 3b, which has been observed in the camera shown in Fig. 3a and c. The camera recorded the view of the marker registered in the near-infrared band 
$(\lambda=850 \mathrm{~nm})$ at $60 \mathrm{fps}$. The IR heater construction was discussed in [17-19]. The created measuring system has enabled the powering of the coil of the woofer with DC voltage (Fig. 2, switch in position I) and also alternating voltage (Fig. 2, switch in position II). DC voltage was used to determine the range and direction of the deflection of the membrane in the function of the value of applied control voltage, which forms the electrodynamic force of the voice coil.

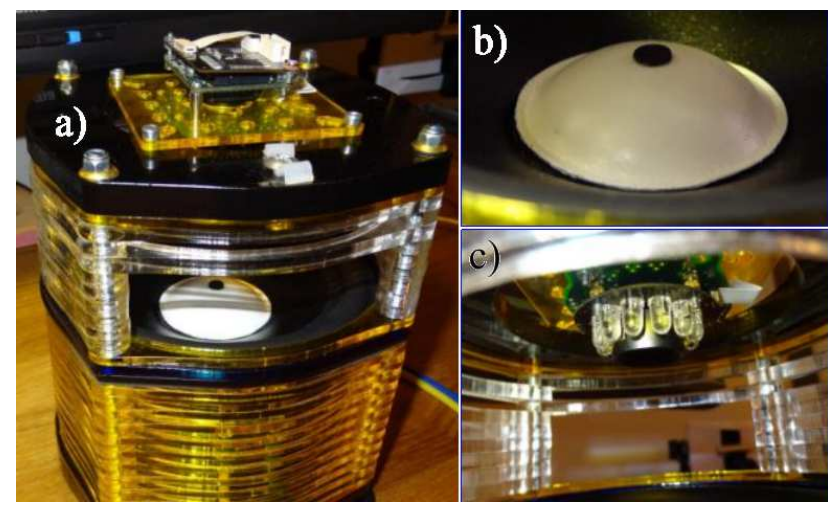

Fig. 3. The construction of the model developed for determining membrane displacement: the model with a mounted camera (a), view of the marker (b), lens and IR heater (c).

The vibration frequency of the membrane was measured for alternating voltages of a known signal shape and amplitude as well as a fixed frequency. Alternating voltage was generated by an AFG 3021 arbitrary generator. The generator was connected to the woofer through a VM 100 amplifier. The tests were carried out, forcing the membrane displacement by administering an alternating sinusoidal signal with an amplitude close to $12 \mathrm{~V}$ and a $f_{\text {set }}$ frequency with a value of $0.5,0.7,0.9,1.0,1.1$, $1.3,1.5,2.0,2.5,3.0,3.5,4.0,5.0$, and $6.0 \mathrm{~Hz}$.

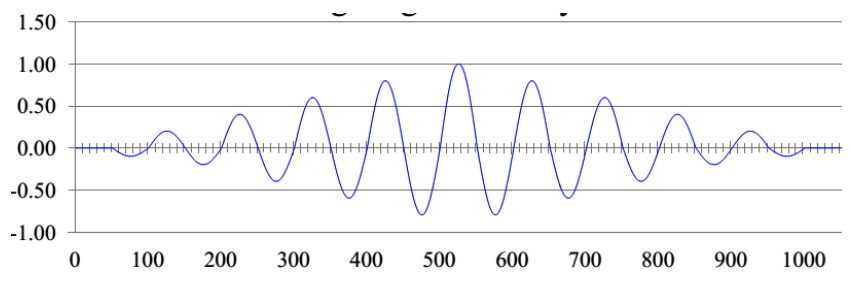

Fig. 4. A sample of the course of voltage generated by an AFG 3021 generator.

Tests for signal paths with a shape as presented in Fig. 4 were performed for the given system configuration. The signal was generated with a frequency equal to 0.5 , $0.7,0.9,1.0,1.1,1.3,1.5,2.0$, and $2.5 \mathrm{~Hz}$. The aim of this study was to examine the possibility of determining the parameters of membrane displacement in conditions of changes in the amplitude of the control signal.

\section{Description of experiments and results of research}

\subsection{Measuring the membrane vibration frequency}

The study was carried out in the setup shown in Fig. 2, in which the source switch of the signal is set to position II. The sinusoidal wave $U_{\text {in }}$ was given at the input of the amplifier with a frequency of $f_{\text {set }}$. The amplitude of the wave form was selected so that the voltage at the output of the amplifier was within the range of $\pm 5.8 \mathrm{~V}$. The membrane moved in the range from $-3.4 \mathrm{~mm}$ to $+3.8 \mathrm{~mm}$ relative to the position established by the lower and upper spider of the speaker in the absence of voltage control. The dependence of the position of the membrane in the function of the voltage control value is presented in Fig. 5. No significant changes were observed in the study on the position of the membrane resulting from the occurrence of the hysteresis phenomenon. The influence of the amplitude of the alternating voltage on the deflection of the membrane was measured three times. The multiple measurements were repeated to reduce the impact of random errors resulting from the use of a highly sensitive (of $0.01 \mathrm{~mm}$ accuracy) dial gauge. The signal resulting from the vibration of the membrane was obtained using the technique presented in [16]. This technique requires the use of a marker. A black marker was used in the study, in the shape of a circle with a diameter of $7 \mathrm{~mm}$, Fig. 3b. For the given marker size the measurement step equalled 0.0048 ( $1 /$ No. of pixels registered for the marker view).

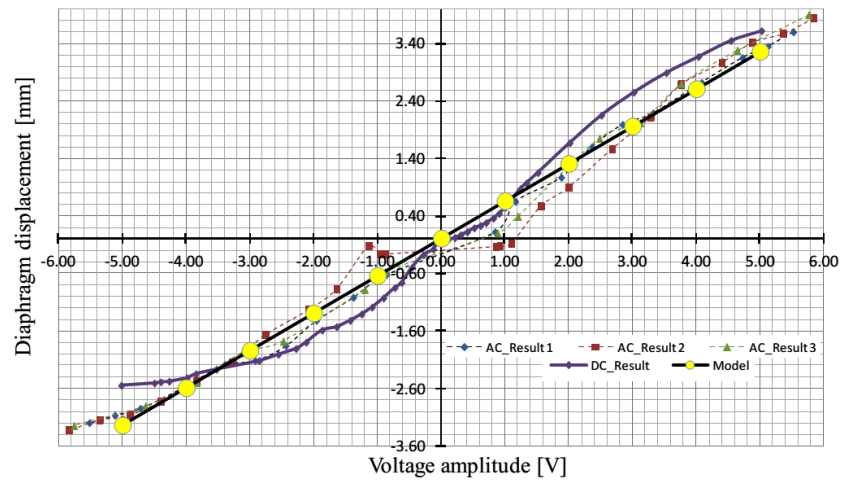

Fig. 5. Speaker membrane displacement as a function of the voltage control amplitude.

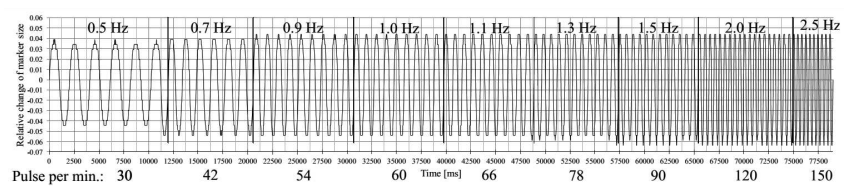

Fig. 6. Changes in marker size recorded for the given frequencies.

Measuring the membrane vibration frequency involved signal analysis, whose shape reflects the changes in the marker size in the function of time. These changes were expressed relative to the size of the marker recorded in 
the rest position. The result obtained for the $f_{\text {set }}$ frequency signal from $0.5 \mathrm{~Hz}$ to $2.5 \mathrm{~Hz}$ is presented in Fig. 6.

Determined relative changes in the size of the marker reflect the nature of the periodic signal, which forces movement on the membrane. The recovered signal, such as the excitation signal, has a periodic nature, and the amplitude change takes on repetitive values within each of the tested frequency bands. A slight change in the size of the marker, depending on the deflection of the membrane - Fig. 6 , is a result of parameters amplifier used, including its bandwidth.
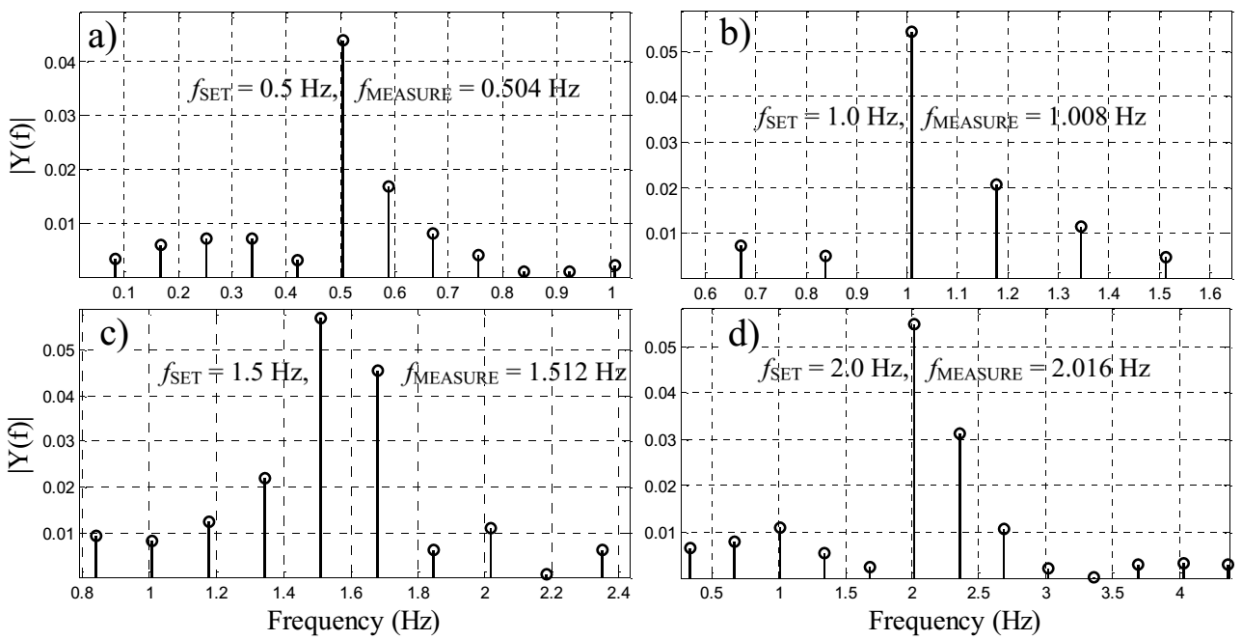

Fig. 7. Examples of signals of amplitude spectra: $f_{\text {set }}$ - inflicted vibration frequency, $f_{\text {measure }}$ - measured vibration frequency.
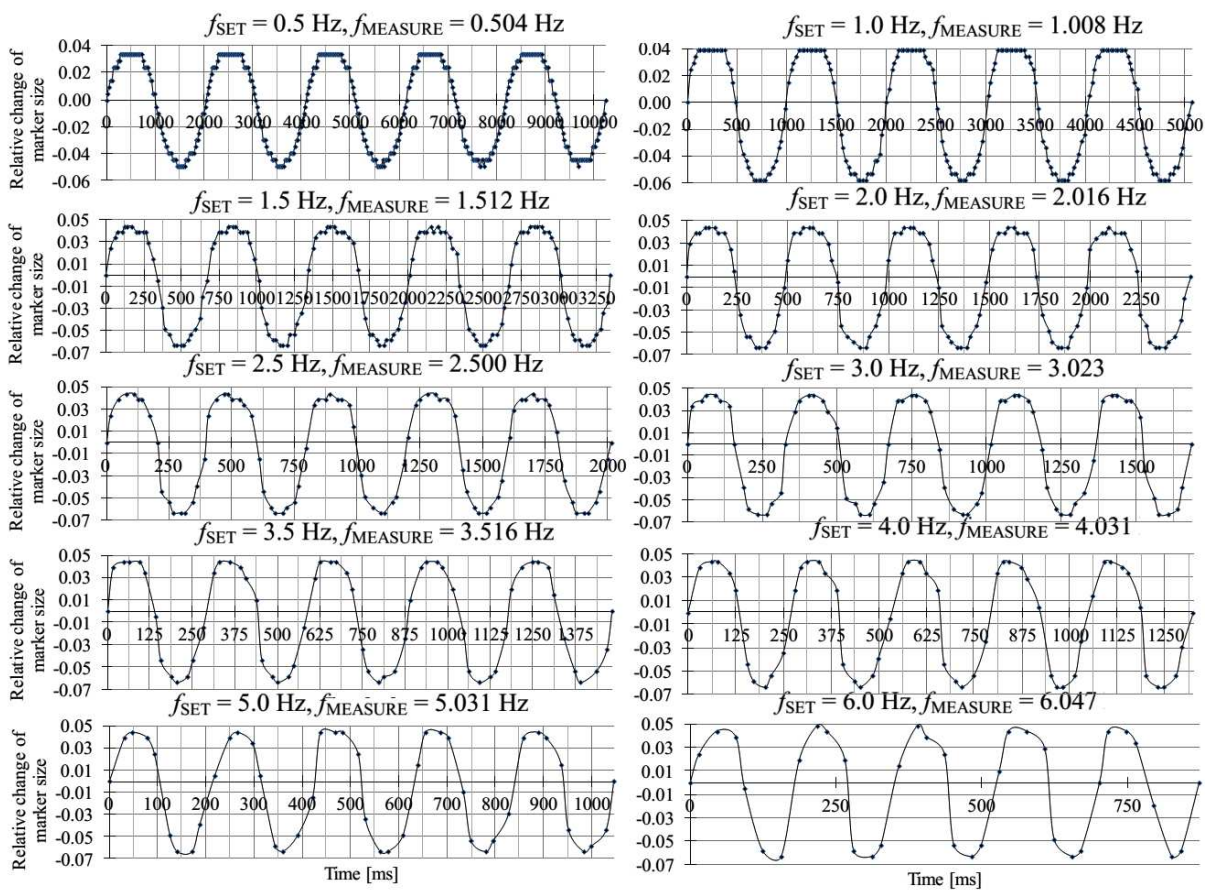

Fig. 8. Result of recorded data: $f_{\text {set }}$ - inflicted vibration frequency, $f_{\text {measure }}$ - measured vibration frequency.

The vibration frequency of the membrane was determined by using a fast Fourier transform (FFT). The sequences of changes in marker size remembered for each input signal underwent analysis, Fig. 8. For each of them the amplitude spectrum of the FFT signal has been computed. Next, the frequency component with a maximum value was searched. This frequency component determines the dominant frequency present in the analysed signal, Fig. 7. The result of the recorded data along with the established $f_{\text {set }}$ and the determined $f_{\text {measure }}$ membrane vibration frequency is presented in Fig. 8. The length of the sequence in the study was chosen, 
so that the frequency measurement error using the FFT technique was less than $1 \%$. The resulting average percentage error of measuring the membrane vibration frequency equalled $0.654 \%$.

\subsection{Measurement of membrane displacement}

The displacement of the membrane is measured using the technique presented in [16]. The measurement was preceded by the calibration procedure, which is performed only once. The purpose of calibration is to determine the dependency between the displacement of membrane $y$ expressed in $\mathrm{mm}$ and marked with an $x$ marker of relative size. The calibration procedure is carried out in two stages. The first stage is to determine the deflection of the membrane in the function of voltage control. The dependence of the membrane deviation on the amplitude of the DC and AC voltage control is presented in Fig. 5. A regression line was determined in the form of function $y(x)=a x+b$ for the carried out measurements. The regression line expresses the dependence of the deflection of the membrane from the value of the control signal.

The second calibration stage is to record changes in the relative size of the marker for the established voltage values. The study was conducted for alternating voltages with fixed $U_{\mathrm{PP}}$ and frequencies of $0.5,1.0,1.5 \mathrm{~Hz}$ and $2.0 \mathrm{~Hz}$. Changes in the size of the marker as well as their averaged values is presented in Fig. 9.

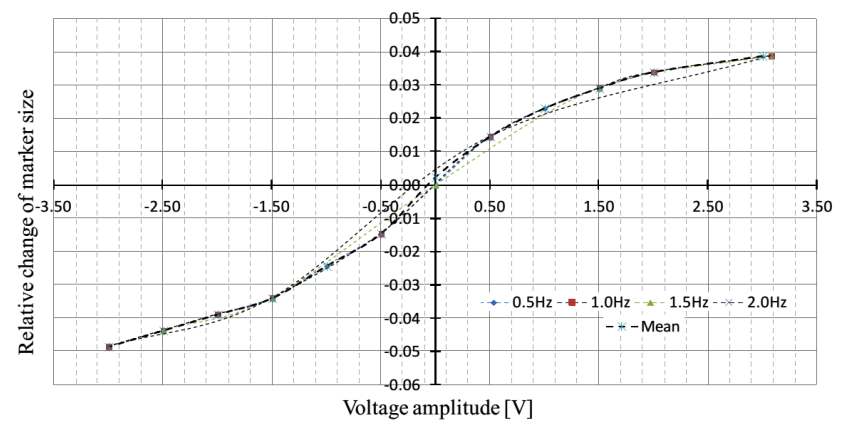

Fig. 9. Changing the size of the marker observed in the function of change of the amplitude of the control voltage.

Characteristics shown in Fig. 9 is described by model $M\left(V, \mu, \sigma, x_{m}\right)$ in the form of

$$
\begin{aligned}
& M\left(V, \mu, \sigma, x_{m}\right)=v_{8} z^{8}+v_{7} z^{7}+v_{6} z^{6}+v_{5} z^{5}+v_{4} z^{4} \\
& \quad+v_{3} z^{3}+v_{2} z^{2}+v_{1} z^{1}+v 0,
\end{aligned}
$$

for which the average value of measurements are: $\mu=0.0056$; the standard deviation of the measured values $\sigma=0.0324 ; x_{m}$ - the measured relative change in the marker size; $z=\left(x_{m}-\mu\right) / \sigma ; V=$ $\left\langle v_{8}, v_{7}, v_{6}, v_{5}, v_{4}, v_{3}, v_{2}, v_{1}, v_{0}\right\rangle$. The determined parameter values of the model equalled: $v_{8}=0.5029, v_{7}=$ $0.1144, v_{6}=-1.6108, v_{5}=-0.2557, v_{4}=1.6544$, $v_{3}=0.7893, v_{2}=-0.6164, v_{1}=0.9553, v_{0}=-0.1861$.
Model $M\left(V, \mu, \sigma, x_{m}\right)$ was used jointly with a simple regression $y(x)=0.6504 x+0.0102$ (Fig. 5), in which $y(x)$ indicated the displacement of the membrane expressed in $\mathrm{mm}$ and $x$ - the voltage control amplitude. As a result the dependences occurring between the measured relative marker size and the membrane displacement are expressed in mm, Fig. 10.

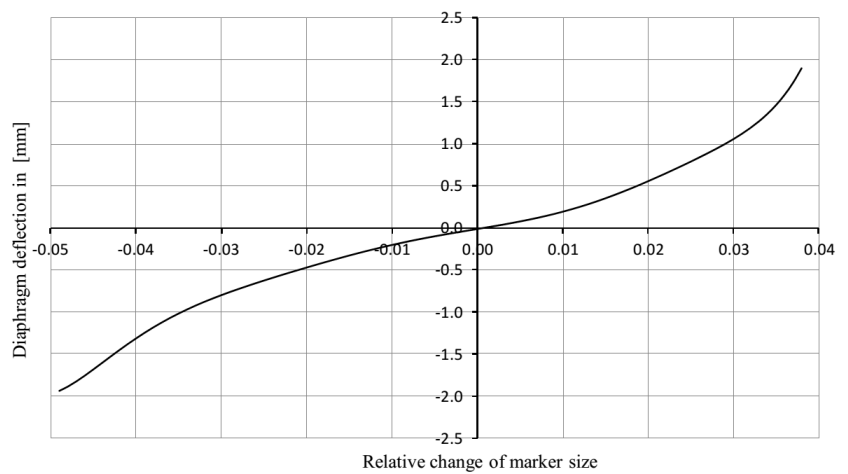

Fig. 10. Change in membrane position in the function of the determined marker size.

This dependence is described by model $\mathrm{M}_{1}\left(V, \mu, \quad \sigma, x_{m}\right)$ of a form (1), for which $\mu=-0.0055 ; \sigma=0.025547 ; v_{8}=0.046881$, $v_{7}=0.015934, v_{6}=-0.24341, v_{5}=-0.058505$, $v_{4}=0.40572, v_{3}=0.25788, v_{2}=-0.24199$ $v_{1}=0.48433, v=-0.10785$, where $x_{m}$ is the measured marker size. Determining the parameters of model $M_{1}\left(V, \mu, \sigma, x_{m}\right)$ concludes the calibration procedure.

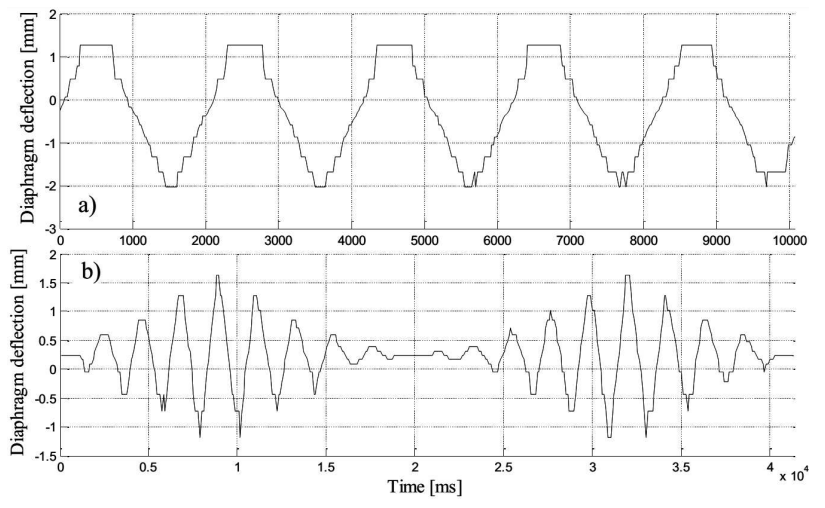

Fig. 11. Membrane displacement determined using the visual technique.

Measuring the membrane displacement is carried out indirectly by measuring the size of the marker. The displacement value is calculated from the model $M_{1}\left(V, \mu, \sigma, x_{m}\right)$ assuming $x_{m}$ to be the relative marker size obtained during measurement. By using the model and techniques presented in [16], it was possible to determine the value and direction of the membrane displacement, relative to the position of the camera, based on information achieved from a single image frame. In the 
setup in Fig. 2 the displacement of the membrane was determined in a time of less than $16 \mathrm{~ms}$. Examples of the results obtained through sequence analysis of images are shown in Fig. 11. In the presented case the voice coil was controlled with a voltage with an amplitude in the range of $\pm 2.5 \mathrm{~V}$. The membrane vibration diagram presented in Fig. 11a was achieved for a sinusoidal input signal with a frequency of $0.5 \mathrm{~Hz}$. The graph presented in Fig. 11b was created as a result of controlling the voltage of the voice coil, whose amplitude was changed in accordance with the pattern shown in Fig. 4. The frequency parameters of the input signal are presented in Fig. 11b.

\section{Conclusions}

The conducted experiments have shown that a motionless camera equipped with a lens with a fixed focal length, using the image processing technique [16], allows determining the displacement of the membrane relative to the position of camera on the basis of a single image. The measurement of the vibration frequency of the membrane is possible based on the analysis of a series of images.

A camera with a resolution of $640 \times 480$ pixels and $60 \mathrm{fps}$ was used in the study. The camera parameters allowed measuring the vibration frequency of the membrane in the range of $6 \mathrm{~Hz}$ and amplitudes of vibrations of the membrane in the entire deflection range.

The accuracy of measuring the movement of the membrane in the presented technique is directly proportional to the image resolution. Frequency measurement accuracy increases with the increase in the length of recorded image sequence. The measured frequency range depends on the number of acquired and analyzed frames per second (FPS).

\section{Acknowledgments}

The author would like to thank Prof. Tadeusz Pustelny as well as Ph.D. Eng. Grzegorz Konieczny from the Silesian University of Technology, whose publications have been an inspiration for this work.

The work was presented at the 8th Conference Integrated Optics - Sensors, Sensing Structures and Methods, IOS'2014 sponsored by Polish Academy of Sciences. The conference was organized by the Committee of Electronics and Telecommunication at the Polish Academy of Sciences in cooperation with the Upper Silesian Division of the Polish Acoustical Society and Photonic Society of Poland, as well as the Department of Optoelectronics at the Silesian University of Technology.

\section{References}

[1] VEGA Americas, Inc., Technologies, Solutions, and Applications - Pressure Measurement, 2014.

[2] WIKA Instrument Corporation, WIKA Handbook Pressure $\mathcal{G}$ Temperature Measurement U.S. Edition, 2014.

[3] G. Konieczny, Z. Opilski, T. Pustelny, E. Maciak, Acta Phys. Pol. A 116, 344 (2009).

[4] T. Martynkien, M. Szpulak, G. Statkiewicz, G. Golojuch, J. Olszewski, W. Urbanczyk, J. Wojcik, P. Mergo, M. Makara, T. Nasilowski, F. Berghmans, H. Thienpont, Opt. Quant. Electron. 39, 481 (2007).

[5] T. Martynkien, G. Statkiewicz, M. Szpulak, J. Olszewski, G. Golojuch, W. Urbanczyk, J. Wojcik, P. Mergo, M. Makara, T. Nasilowski, F. Berghmans, H. Thienpont, Meas. Sci. Technol. 18, 3055 (2007).

[6] G. Konieczny, Z. Opilski, T. Pustelny, Acta Phys. Pol. A 120, 688 (2011).

[7] G. Konieczny, T. Pustelny, Acta Phys. Pol. A 122, 962 (2012).

[8] G. Konieczny, T. Pustelny, P. Marczyński, Acta Phys. Pol. A 124, 479 (2013).

[9] G. Konieczny, T. Pustelny, P. Marczyński, Acta Phys. Pol. A 124, 483 (2013).

[10] A.A. Dorrington, T.W. Jones, P.M. Danehy, R.S. Pappa, AIAA J. 42, 2124 (2004).

[11] S. Binua, V.P. Mahadevan Pillaia, N. Chandrasekaran, Opt. Laser Technol. 39, 1537 (2007).

[12] K. Murawski, Przeglad Elektrotechniczny 9, 184 (2010) (in Polish).

[13] K. Murawski, in: Proc. XV Int. Conf. on Methods and Models in Automation and Robotics (MMAR), Międzyzdroje (Poland), 2010, p. 356.

[14] K. Murawski, R. Różycki, P. Murawski, A. Matyja, M. Rekas, Acta Phys. Pol. A 124, 517 (2013).

[15] K. Murawski, K. Różanowski, Acta Phys. Pol. A 124, 509 (2013).

[16] K. Murawski, Acta Phys. Pol. A 127, p23 (2015).

[17] K. Różanowski, K. Murawski, Acta Phys. Pol. A 122, 874 (2012).

[18] K. Murawski, T. Arciszewski, K. De Jong, Eng. Comput. 16, 275 (2000).

[19] K. Różanowski, K. Murawski, Acta Phys. Pol. A 124, 558 (2013). 